\title{
Inventario de la avifauna reproductora de Jaizkibel y Jaizubia (Gipuzkoa)
}

\author{
Hegazti-fauna ugaltzaileen inbentarioa Jaizkibel eta Jaizubian (Gipuzkoa) \\ Inventory of birds breeding in Jaizkibel and Jaizubia (Gipuzkoa)
}

\author{
Juan Arizaga ${ }^{1 *}$ \\ ${ }^{1}$ Departamento de Ornitología, Sociedad de Ciencias Aranzadi, Zorroagagaina 11, E-20014 Donostia-S. Sebastián. \\ *Correspondencia: jarizaga@aranzadi.eus
}

\section{RESUMEN}

Recientemente se ha puesto de manifiesto la relevancia de Jaizkibel y el complejo húmedo asociado de Jaizubia como una zona de gran riqueza biológica y geológica. El objetivo de este artículo es elaborar un inventario, a través de revisión bibliográfica y observaciones inéditas, de la avifauna reproductora en Jaizkibel y Jaizubia. Sólo se han considerado las especies de nidificación segura, en las que se ha registrado su cría al menos una vez desde 1999 hasta 2012. Se constata la nidificación de 81 especies de aves, lo cual supone un 64,8\% de las especies que se citan para toda la provincia de Gipuzkoa. En cuanto a especies interesantes desde un punto de vista de la conservación, se detectaron 16 incluidas en el catálogo de especies amenazadas de la CAPV. En consecuencia, la riqueza de especies de aves nidificantes en Jaizkibel es alta, probablemente una de las más elevadas de Gipuzkoa para el área de muestreo considerada.

PALABRAS CLAVE: Aves nidificantes, censos, conservación, especies amenazadas, figura de protección, LIC.

\section{LABURPENA}

Berriki Jaizkibel eta berarekin erlazionatutako Jaizubiako hezegune multzoen garrantzia agerian jarri da, gehien bat ikuspuntu biologiko eta geologikotik agertzen duten aberastasuna medio. Artikulu honen helburua Jaizkibel eta Jaizubian hegazti habiagileen inbentario bat sortzea da, lan honetan azterketa bibliografikoa eta argitaragabeko behaketak erabiliko dira. Soilik kontuan hartu dira espezie habiagile ziurrak, hau da 1999tik 2012rarte gutxienez behin ugaldu direnak. 81 hegazti habiagile egiaztatu dira, hau da Gipuzkoan topatu daitezkeenen \%64,8. Kontserbazio egoerari dagokionez EAEko espezie mehatxatuen zerrendan dauden 16 espezie antzeman dira. Ondorioz, Jaizkibelgo hegazti habiagileen aberastasuna altua da, Gipuzkoan topa litekeen altuenetakoa, betiere ikerketa area barneratzen duen eremu osoan aintzat hartu ezkero.

GAKO-HITZAK: Hegazti habiagileak, zentsuak, kontserbazioa, espezie mehatxatuak, babeserako irudia, GKL.

\section{ABSTRACT}

Jaizkibel and the wetlands associated with the Jaizubia stream have been revealed to be an area of high biological and geological interest. The aim of this work is to review the list of the breeding avifauna at these two zones. I have only considered those birds (species) proved to breed in these zones at least once from 1999 to 2012. I detected 81 species overall, and this was a $64.8 \%$ of the avian species found to breed in Gipuzkoa. From the standpoint of species of concern, 16 were included within the Basque list of threatened species. The richness of breeding bird species at Jaizkibel and Jaizubia was high, probably one of the highest in Gipuzkoa for the survey area here considered.

KEY WORDS: Breeding birds, surveys, conservation, threatened species, protected area, SCI.

\section{INTRODUCCIÓN}

La elaboración de inventarios de especies amenazadas es básica en el ámbito del manejo, conservación y protección del patrimonio natural. La existencia de determinadas especies en un paraje dado, y en especial de aves, constituye un criterio ampliamente empleado para su valoración y protección legal. Asimismo, el desarrollo de tales inventarios puede resultar de gran utilidad para determinar la eficacia de la figura de protección de un espacio y/o evaluar la conveniencia de aumentar el área de protección.

La montaña de Jaizkibel se emplaza en el extremo NE de la provincia de Gipuzkoa. Se trata de un monte de $547 \mathrm{~m}$ s.n.m., con dos claras vertientes: (1) la septentrional, compleja, de pendientes suaves en la zona oriental y abruptas en la zona occidental, atravesada de vaguadas y pequeños valles y (2) la meridional, de pendientes pronunciadas, más uniformes que la septentrional. En la zona baja de la ladera meridional de Jaizkibel, además, se localiza el complejo húmedo asociado la regata de Jaizubia.
Respecto a la vegetación actual, la mayor parte de Jaizkibel está actualmente deforestada para su aprovechamiento como pastos para el ganado. Domina en consecuencia (ca. $70 \%$ de la superficie) la vegetación herbácea (pastos, helechales) y el matorral (brezales, etc.). Existe cierto número de monocultivos forestales artificiales (ca. 15\% de la superficie), principalmente plantaciones de pino insignis Pinus radiata D. Don. En determinados puntos, asociado a suelos ácidos, existen pequeñas manchas de robledal de Quercus pirenaica Wild y bosques de especies autóctonas (ca. < 5\% de la superficie). La superficie restante (ca. 10\%) está constituida de roquedos interiores y costeros, playas y turberas. En la zona meridional no incluida en el LIC quedan alisedas (Alnus spp.) y robledales ( $Q$. robur L.) bien conservados, así como una zona de marismas ligada a la regata de Jaizubia, recuperada en 2005 . Esta zona consta de carrizales y llanuras de limo y vegetación halofítica.

Parte de Jaizkibel (2434 ha) se propuso en 2004 como Lugar de Importancia Comunitaria (LIC; decisión 2004/813/CE; código ES2120017). En la actualidad, el LIC 
de Jaizkibel ha de designarse como Zona de Especial Conservación (ZEC). EI LIC de Jaizkibel, no obstante, deja al margen el ámbito marino ligado directamente al macizo y la mayor parte de la ladera meridional, incluido el complejo húmedo asociado a la regata de Jaizubia (este último no obstante incluido en el LIC de Txingudi; código ES2120018) (Fig. 1).

Recientemente se ha puesto de manifiesto la relevancia de Jaizkibel como una zona de gran riqueza biológica y geológica (Arizaga, 2012). Debido a ello, la puesta al día de su valor natural se hace particularmente necesaria en un contexto socioeconómico complejo, donde parte de la sociedad ha propuesto construir un puerto exterior cuya ejecución comprometería la conservación del patrimonio natural de la montaña.

En el presente artículo se pretende actualizar el conocimiento relativo a la avifauna reproductora en Jaizkibel y Jaizubia.

\section{MATERIAL Y MÉTODOS}

El área objeto de estudio se incluye en el área incluida en el LIC de Jaizkibel (Fig. 1). Esta superficie abarca la zona emergida de la montaña, incluida en los municipio de Pasaia (Donibane), Lezo y Hondarribia. Asimismo, se ha incluido en este estudio la ladera meridional de Jaizkibel no incluida en el LIC y el humedal de Jaizubia, incluido en el LIC de Txingudi. El límite de toda el área de estudio se indica en la Fig. 1.

Para la revisión de la avifauna reproductora en Jaizkibel se consideran el inventario (atlas) de aves nidificantes de Gipuzkoa (Aierbe et al., 2001), los resultados del programa de seguimiento de aves comunes de la
CAPV (fuente: IHOBE, Gobierno Vasco-Eusko Jaurlaritza), la base de datos de anillamiento de la Sociedad de Ciencias Aranzadi, informes y observaciones inéditas no registradas de manera formal en ningún catálogo ni publicación. Sólo se han inventariado las especies de nidificación segura, para las que se ha registrado su cría al menos una vez desde 1999 (fecha de censo para el atlas de 2001) en adelante (hasta 2012).

Para cada una de las especies se ha recopilado la siguiente información: (1) hábitat, (2) estima de su tamaño (abundancia) o densidad de la población en Jaizkibel y Jaizubia, si se conoce, y (3) status migratorio en el área de estudio. En cuanto al status migratorio, se han considerado las siguientes categorías: sedentario, migrante presahariano (especies que invernan en la región circummediterránea), migrante transahariano (especies que invernan en África tropical, al sur del Sahara).

\section{RESULTADOS \\ Resultados generales}

En conjunto, se detectaron un total de 81 especies de aves de nidificación segura en Jaizkibel y Jaizubia, lo cual supone un $64,8 \%$ de las especies que se reproducen en Gipuzkoa (125, según el atlas de aves nidificantes de 2003) (Aierbe et al., 2001).

De las especies que se detectaron en Jaizkibel y Jaizubia, 16 de ellas se incluyen en el Catálogo Vasco de Especies Amenazadas de la Fauna y Flora Silvestre y Marina (Orden de 10 de enero de 2011), de las que dos se consideran "Vulnerable" [Neophron percnopterus (L., 1758) y Charadrius dubius Scopoli, 1786], seis "Rara" y ocho de "Interés Especial" (Tabla 1). Neophron percnopterus, ade-

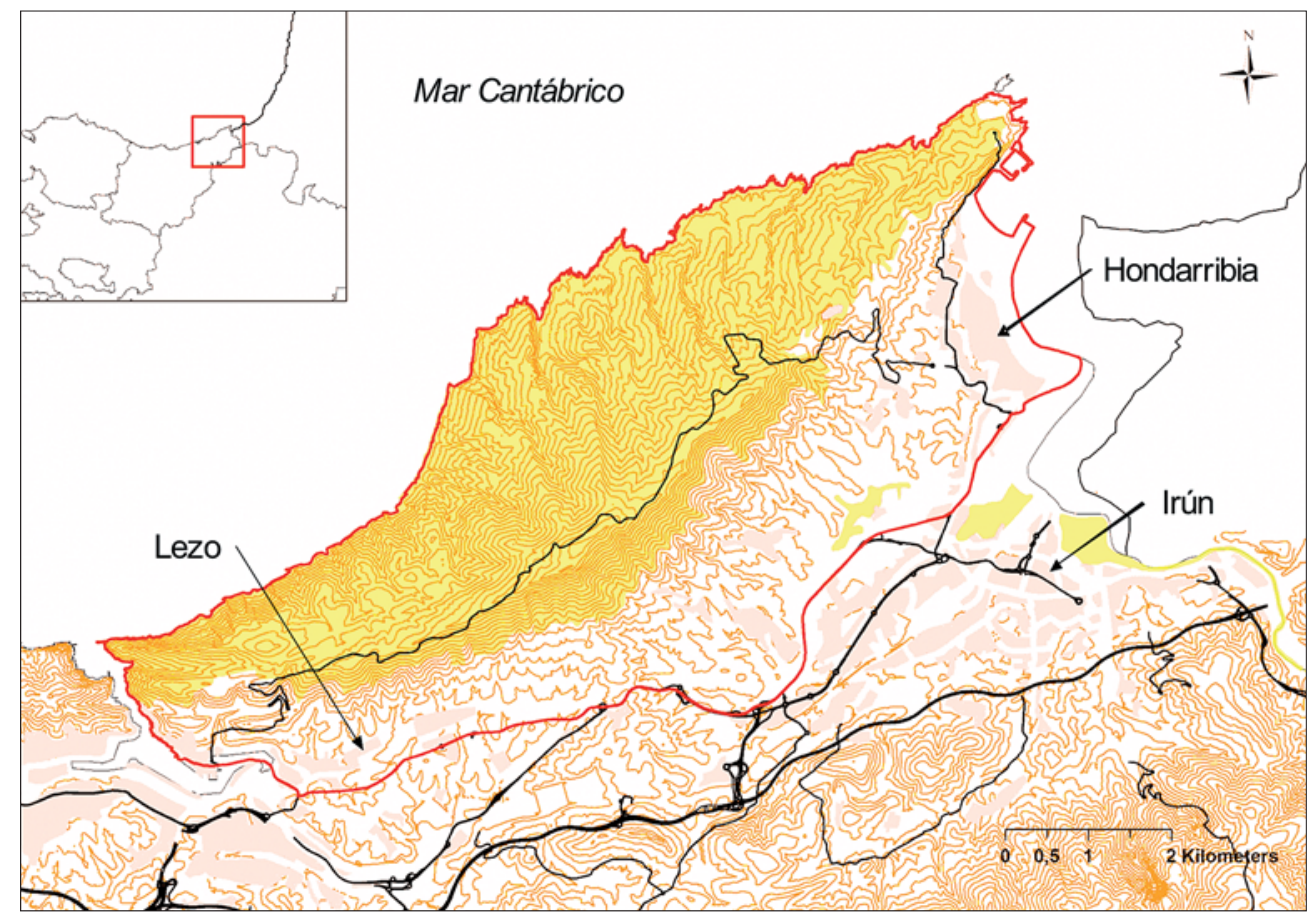

Fig. 1. - Área de estudio en Jaizkibel. El polígono rojo abarca el área de muestreo tenida en cuenta en esta revisión. El área en verde claro es el LIC de Jaizkibel (mancha grande) y la regata de Jaizubia, esta última incluida en e LIC de Txingudi. Las líneas negras son las carreteras principales.

1. Irudia. Jaizkibelgo ikerketa area. Poligono gorriak berrikuspen honetan haintzat hartu den ikerketa area adierazten du. Azalera berde argiak Jaiz kibelgo GKL (orbain handia) eta Jaizubiako ubidea, azken hau Txingudiko GKLn dagoena. Errepide nagusiak lerro beltzez adierazi dira. 


\begin{tabular}{|l|c|c|c|}
\hline Especie & CAPV & España & $\begin{array}{c}\text { Directiva Aves } \\
\text { (Anexo I) }\end{array}$ \\
\hline Nachybaptus ruficollis & RA & & Si \\
\hline Circaetus gallicus & VU & EN & Si \\
\hline Circus cyaneus & RA & & Si \\
\hline Falco subbuteo & IE & & Si \\
\hline Falco peregrinus & RA & NT & \\
\hline Rallus aquaticus & RA & & \\
\hline Charadrius dubius & RA & & Si \\
\hline Caprimulgus europaeus & VU & & Si \\
\hline Alcedo atthis & IE & & \\
\hline Jynx torquilla & $\mathrm{IE}$ & NT & \\
\hline Dendrocopos minor & $\mathrm{IE}$ & & \\
\hline Monticola solitarius & $\mathrm{IE}$ & & $\mathrm{Si}$ \\
\hline Acrocephalus scirpaceus & $\mathrm{IE}$ & & $\mathrm{Si}$ \\
\hline Sylvia undata & $\mathrm{RA}$ & & \\
\hline Lanius collurio & & & \\
\hline Corvus corax & & & \\
\hline Coccothraustes coccothraustes & $\mathrm{IE}$ & & \\
\hline
\end{tabular}

CAPV. Orden de 10 de enero de 2011. VU: Vulnerable; RA, Raro; IE, Interés Especial. España. Libro Rojo de las Aves de España (Madroño et al., 2004). EN: En Peligro; NT, Casi Amenazado.

Tabla 1. - Listado de especies y su status de conservación en la CAPV y en España. Se considera, además, su inclusión en el Anexo I de la Directiva Aves. Las especies no incluidas en ningún catálogo de especies amenazadas se excluyen de este listado.

1. Taula. Espezie zerrenda eta EAEn eta Espainian agertzen duen kontserbazio egoera. Horrez gain Hegaztien Zuzentarauan I. eranskinean dauden espezieak adierazten dira. Espezie mehatxatuen katalogoetan ez dauden espezieak zerrenda honetatik kanpo geratu dira.

más, también se incluye como especie "En Peligro" en el Libro Rojo de las Aves de España (Madroño et al., 2004). Asimismo, ocho especies se incluyen en el Anexo I de la Directiva Aves (Tabla 1).

\section{Listado de especies de reproducción segura}

Las especies han sido ordenadas según los criterios de Clavell et al. (2005).

Tachybaptus ruficollis (Pallas, 1764). Nidifica en las lagunas del ámbito de Jaizubia tras la recuperación de la marisma en 2005. El número máximo de parejas observadas durante el periodo 2010-2012 es de 7 (M. Etxaniz, com. per.). Sedentario.

Anas platyrhynchos L., 1758. Nidifica en las lagunas del ámbito de Jaizubia, así como los tramos inferiores de algunas regatas de la ladera meridional de Jaizkibel y las charcas del campo de golf (J. Belzunce, com. per.). Sedentario.

Anas strepera L., 1758. Cría en las lagunas del ámbito de Jaizubia (laguna de S. Joaquín). 1 ó 2 parejas reproductoras en 2009, 2010 y 2011 (J. Belzunce, com. per.). Sedentario.

Cygnus olor Gmelin, 1789. Se cita su cría (1 pareja) en la laguna de S. Joaquín (Jaizubia) en 2012 (J. Belzunce, com. per.). Sedentario.

Neophron percnopterus. En Jaizkibel se constata la nidificación de 1 pareja a partir de 2009 (T. Aierbe, M. Olano, com. per.), en un acantilado de la ladera orientada al mar. Transahariano.
Circaetus gallicus (Gmelin, 1788). Se constata la nidificación de 1 pareja a partir de 2005, en un pino localizado en la ladera orientada al mar (T. Aierbe, M. Olano, com. per.), si bien la especie se observa en Jaizkibel, durante e periodo de cría, ya en la década de 1990 (Aierbe et al., 2001). Transahariano.

Circus cyaneus (L., 1766). Se cita la cría de 1 pareja en 2001 y 2002 (J. Belzunce, com. per.). Posteriormente no se ha constatado su reproducción. Presahariano.

Buteo buteo (L., 1758). Nidifica en arbolado. Presente en Jaizkibel (Aierbe et al., 2001). Asociado a campiña o plantaciones de especies arbóreas tanto exóticas como nativas. No obstante, en 2011 y 2012 se localiza una pareja que cría en un roquedo en la ladera septentrional (J. Belzunce, com. per.). Un mínimo de 6 parejas reproductoras (J. Belzunce, com. per.). Sedentario.

Falco tinnunculus L., 1758. Cría en Jaizkibel, normalmente, asociado a roquedos (Aierbe et al., 2001). Un mínimo de 1 pareja reproductora. Presente en la ladera septentrional. Sedentario.

Falco subbuteo L., 1758. Se observa en época de cría a partir de 2000, si bien sólo en 2010 se constata la nidificación de 1 pareja (se observan pollos volantones) en un pino de la ladera meridional (J. Belzunce, com. per.). Transahariano.

Falco peregrinus Tunstall, 1771. Cría en roquedos (Aierbe et al., 2001) en la ladera que da al mar. En 2012 se citan un total de 3 parejas (T. Aierbe, M. Olano, com. per.), si bien durante los últimos años ha nidificado en la zona de manera habitual (Álvarez et al., 2005). Sedentario.

Rallus aquaticus L., 1758. Cría en carrizales en Jaizubia; en 2007 se localiza una pareja con pollos (J. Belzunce, com. per.). Sedentario.

Gallinula chloropus (L., 1758). Cría en las lagunas del ámbito de Jaizubia, tramos inferiores de algunas regatas de la ladera meridional y en las charcas del campo de golf de Hondarribia (J. Belzunce, com. per.). Sedentario.

Fulica atra L., 1758. Cría en las lagunas del ámbito de Jaizubia, a partir de 2005 (J. Belzunce, com. per.). Sedentario.

Scolopax rusticola L., 1758. Cría en bosques. En mayo de 2002 se localiza un ejemplar saliendo de un nido en el municipio de Hondarribia (D. Calleja, com. per.). Posteriormente se comprobó cómo el nido había sido abandonado cuando tenía un huevo. Se atribuye la presencia de perros de caza como motivo de este abandono. En Gipuzkoa, la especie es, aparentemente, sedentaria.

Charadrius dubius. Cría en espacios llanos arenosos o pedregosos, cerca o junto al agua. Cría (1 pareja) en Jaizubia a partir de 2009 (J. Belzunce, com. per.). Transahariano.

Larus michahellis Naumann, 1840. Cría en roquedos costeros en Turuia y, esporádicamente (i.e., en determinados años) en el islote de Amuitz frente al Cabo de Higuer (Aierbe et al., 2001). En el censo de 2007 (el último) se constata una colonia con 45 parejas (Arizaga et al., 
2009). El tamaño de la colonia alcanzó un máximo de 83 parejas en 1990, tras lo cual ha sufrido un declive progresivo. Sedentario.

Streptopelia decaocto (Frivaldszky, 1838). Cría en arbolado, asociado al núcleo urbano de Hondarribia (Aierbe et al., 2001). Igualmente, habitual en el medio rural (J. Arizaga, obs. per.). Sendentario.

Cuculus canorus L., 1758. Parasita nidos de otras especies de aves (paseriformes), en todo tipo de hábitats. En 1999 se observaron pollos volantones (Aierbe et al., 2001). En 2007 un pollo es alimentado en un nido de Troglodytes troglodytes L., 1758 en las proximidades del caserío Justiz (J. Belzunce, com. per.). Transahariano.

Tyto alba Scopoli, 1769. Asociado a construcciones en áreas de campiña, principalmente (Aierbe et al., 2001). Número de parejas no estimado. Sedentario.

Otus scops (L., 1758). Nidifica en huecos de árboles en campiña, principalmente (Aierbe et al., 2001). Número de parejas no conocido. En la década de 1990, la recogida de pollos volantones provenientes de Hondarribia es habitual en el centro de recuperación de Arranoetxea (Aierbe et al., 2001). A partir de entonces se comprueba una regresión significativa (J. Belzunce, com. per.). Transahariano.

Strix aluco L., 1758. Nidifica en arbolado. En Jaizkibel es habitual (Aierbe et al., 2001). En la década de 1990 se cita la recogida en el centro de recuperación de Arranoetxea de una cría que proviene de la zona de Hondarribia (Aierbe et al., 2001). En 2008 un volantón es localizado al ser acosado por pajarillos en Leureta (J. Belzunce, com. per.). Sedentario.

Caprimulgus europaeus L., 1758. Nidifica en el suelo en zonas de landa o arbolado. En Jaizkibel se cita como reproductor seguro en 2000, cuando el 08.07.2000 se ven dos volantones en la ladera meridional (D. Calleja \& M. Guereñu, com. per.). Transahariano.

Apus apus (L., 1758). Cría en construcciones. En Jaizkibel presente en núcleos urbanos, como el de Hondarribia (Aierbe et al., 2001). Transahariano.

Alcedo atthis (L., 1758). Ligado a cursos de agua. Se constata la nidificación de 1 pareja en Jaizubia en 2008 y 2009 (J. Belzunce, com. per.). Sedentario.

Jynx torquilla L., 1758. Nidifica en huecos de árboles. En Jaizkibel se cita la cría en caja-nido, en la zona del golf (J. Arizaga, obs. per.). Presente en zonas de arbolado disperso y en campiña (J. Belzunce, com. per.). Transahariano.

Picus viridis (L., 1758). Cría en árboles, principalmente arbolado disperso, si bien también se observa en bosques compactos (J. Belzunce, com. per.). Sedentario.

Dendrocopos major (L., 1758). Nidifica en árboles. Asociado en Jaizkibel a manchas de campiña y arbolado, tanto frondosas como coníferas (Aierbe et al., 2001; J. Belzunce, com. per.). Sedentario.

Dendrocopos minor (L., 1758). Cría en bosque de ribera, fundamentalmente ligados a regatas de la ladera meridional de Jaizkibel, si bien también cría en la ladera que da al mar (e.g., la regata de Justiz) (J. Belzunce, obs. per.). Sedentario.

Alauda arvensis L., 1758. Cría en pastizales cercanos a la cima de Jaizkibel. Mínimo, 2-3 parejas (J. Belzunce, com. per.). Aparentemente sedentario.

Hirundo rustica L., 1758. Cría en caseríos y fincas aisladas, en zonas de campiña (Aierbe et al., 2001). Transahariano.

Delichon urbica (L., 1758). Ligado a construcciones en el núcleo urbano de Hondarribia (Aierbe et al., 2001), Lezo (L. Romero, com. per.). Transahariano.

Anthus trivialis L., 1758. Cría en arbolado disperso en espacios abiertos. Se cita en brezales con arbolado disperso (Aierbe et al., 2001; J. Belzunce, com. per.). Transahariano.

Motacilla alba L., 1758. Cría en proximidades de caseríos y núcleos urbanos (J. Belzunce, com. per.). Sedentario.

Motacilla cinerea. Cría en algunas regatas de las dos laderas (J. Belzunce, com. per.). Sedentaria.

Motacilla flava L., 1758. Cría en pastizales de carácter húmedo. En 2006 se cita 1 pareja en la laguna de S. Joaquín (J. Belzunce, com. per.). Transahariano.

Troglodytes troglodytes L., 1758. Cría en vegetación baja, generalmente ligado a zarzas, etc. Presente en Jaizkibel (Aierbe et al., 2001), en prácticamente todo tipo de hábitats (arbolado, brezales, etc.). Sedentario.

Prunella modularis (L., 1758). Cría en matorral. En Jaizkibel se cita en brezales y manchas de argomal (Aierbe et al., 2001). Aparentemente sedentario.

Erithacus rubecula (L., 1758). Cría en vegetación baja, en todo tipo de hábitats (Aierbe et al., 2001). Posiblemente sedentario.

Phoenicurus ochruros (Gmelin, 1774). Asociado a roquedos y construcciones. Presente en Jaizkibel (Aierbe et al., 2001). Posiblemente sedentario.

Saxicola torquata (L., 1766). Asociado a matorral y campiñas, requiere de espacios abiertos para alimentarse. En Jaizkibel ligado, principalmente, a brezales y manchas de argomal (Aierbe et al., 2001). Sedentario.

Monticola solitarius (L., 1758). Cría en roquedos en la ladera que da al mar (Aierbe et al., 2001). Sedentario.

Turdus merula L., 1758. Asociado a todo tipo de hábitats con arbolado o arbustos con arbolado disperso (Aierbe et al., 2001). Aparentemente sedentario,

Turdus philomelos Brehm, 1831. Asociado a bosques y manchas de arbolado (Aierbe et al., 2001). Sedentario.

Cisticola juncidis L., 1758. Asociado a pastizales de hierba alta, donde ubica su nido. Observado en campiñas de la ladera septentrional (J. Arizaga, obs. per.). En invierno se desplaza hacia zonas bajas, ocupando carrizales de marismas como la de Jaizubia (Mendiburu et al., 2009; Mendiburu et al., 2010). 
Locustella naevia (Boddaert, 1783). Asociado a manchas de argomal, principalmente. Es fotografiada en Jaizkibel durante el periodo de cría, en canto de celo (J. M. Grandío, com. per.). Transahariano.

Sylvia undata (Boddaert, 1783). Asociada a manchas de argomal y brezales (Aierbe et al., 2001). Sedentario. Se trata de una especie de interés por su asociación con ambientes de carácter mediterráneo.

Sylvia borin (Boddaert, 1783). Asociado a arbolado, cría en arbustos y zarzas densos. Se observa en la campiña de la ladera meridional y algunas regatas de la ladera que da al mar (J. Belzunce, com. per.). Transahariano.

Sylvia atricapilla (L., 1758). Cría a baja altura en zarzas o similar en general en zonas umbrías junto al agua (Aierbe et al., 2001), pasa el invierno posiblemente en la región mediterránea (Tellería et al., 1999).

Cettia cetti L., 1758. Cría en zonas junto al agua. Principalmente en el ámbito de Jaizubia, si bien algunas parejas también se reproducen en algunas regatas de la ladera septentrional (J. Belzunce, com. per.). Tras el periodo de cría es habitual en carrizales como el de Jaizubia (Mendiburu et al., 2009; Mendiburu et al., 2010). Sedentario.

Acrocephalus scirpaceus (Hermann, 1804). Cría en carrizales en Jaizubia (J. Arizaga, obs. per.). Transahariano.

Hippolais polyglotta (Vieillot, 1817). Asociado a arbustos y matorral en todo el ámbito de Jaizkibel (J. Belzunce, com. per.). Transahariano.

Phylloscopus ibericus/collybita. Cría en masas arboladas tanto de frondosas como coníferas. La mayor parte de los ejemplares se consideran $P$. ibericus (J. Belzunce, com. per.). P. ibericus posiblemente pasa el invierno en África subsahariana.

Regulus ignicapillus (Temminck, 1820). Ligado a monocultivos de pino y otro tipo de coníferas, se cita asimismo en el núcleo urbano de Hondarribia (Aierbe et al., 2001). Posiblemente sedentario, aunque no comprobado.

Muscicapa striata (Pallas, 1764). Nidifica en árboles. Se cita en Hondarribia (el 30.06.2002 se localiza un nido con cuatro huevos en una maceta del entorno de campiña del municipio; D. Calleja \& M. Guereñu, com. per.) y en el golf (el 30.06.2003, se localiza un joven junto a un adulto; D. Calleja \& M. Guereñu, com. per.). Transahariano.

Aegithalos caudatus (L., 1758). Cría en arbolado, generalmente en ecotonos de campiñas, líneas de seto, etc. (Aierbe et al., 2001). Sedentario.

Lophophanes cristatus (L., 1758). Cría en arbolado. En Jaizkibel se cita en robledal, plantación de pino y bosque mixto (Aierbe et al., 2001). Sedentario.

Poecile palustris (L., 1758). Cría en robledales, es habitual en la ladera meridional, principalmente en la zona del golf, donde se observa incluso en caja-nido (J. Arizaga, obs. per.), y proximidades de Jaizubia (J. Belzunce, com. per.). Sedentario.
Parus ater (L., 1758). Ligado a monocultivos de pino, principalmente (Aierbe et al., 2001). Sedentario.

Cyanistes caeruleus (L., 1758). Cría en huecos en arbolado de todo tipo, principalmente en caducifolios. Citado en paisaje de campiña (Aierbe et al., 2001) Sedentario.

Parus major L., 1758. Cría en huecos en arbolado de todo tipo. Citado en paisaje de campiña (Aierbe et al. 2001). Sedentario.

Sitta europea L., 1758. Cría en huecos, generalmente ligado a robledales de la ladera meridional (Aierbe et al., 2001). Sedentario.

Certhia brachydactyla Brehm, 1820. Ligado a arbolado, en todo tipo de bosques e incluso en seto bien desarrollado (Aierbe et al., 2001). Sedentario.

Lanius collurio L., 1758. Cría en setos de carácter espinoso, fundamentalmente en zarzas. Citado en Jaizkibe en paisaje de campiña (Aierbe et al., 2001), al cual está estrechamente ligado (Tucker \& Heath, 2004). Tambien se cita en brezales/argomales abiertos en la ladera septentrional (J. Belzunce, com. per.). Transahariano.

Garrulus glandarius (L., 1758). Asociado a arbolado (Aierbe et al., 2001). Sedentario.

Pica pica (L., 1758). Cría en zonas de arbolado, en todo tipo de ambientes salvo zonas de bosque. Cría segura al menos desde el 2005 (J. Belzunce, com. per.) en Jaizubia y el entorno de campiña de Hondarribia. En 2011 se constata, en una urbanización del entorno de campiña de Hondarribia, una pareja con un mínimo de tres pollos del año (M. Guereñu, com. per.). Sedentario.

Corvus corone L., 1758. Cría ligado a arbolado en campiñas de la ladera meridional y junto a zonas de pastos de la ladera que da al mar (J. Belzunce, com. per.). Sedentario.

Corvus corax L., 1758. Nidifica en roquedos (Aierbe et al., 2001), en la ladera que da al mar (J. Belzunce, com. per.). El número de parejas se estima en 3-4. Sedentario.

Sturnus vulgaris L., 1758. Ligado a robledales en campiña (J. Belzunce, com. per.). Probablemente sedentario.

Oriolus oriolus L., 1758. Cría de manera esporádica en robledales y otro tipo de arbolado mixto en el ámbito de Jaizubia (J. Belzunce, com. per.). Transahariano.

Passer domesticus (L., 1758). Presente en núcleos urbanos y también en caseríos y paisaje de campiña (Aierbe et al., 2001). Sedentario.

Passer montanus (L., 1758). Ligado al paisaje de campiña. Durante el periodo de cría, se anilla periódicamente en la regata de Jaizubia proveniente de las campiñas del entorno. Sedentario.

Fringilla coelebs L., 1758. Asociado a arbolado de caducifolios (Aierbe et al., 2001). Posiblemente sedentario.

Serinus serinus (L., 1766). Cría en arbolado, en campiñas y ecotonos de bosque de caducifolios, también en 
árboles dispersos en argomales, brezales, etc. (Aierbe et al., 2001). Status migratorio no conocido (en Urdaibai, unos $100 \mathrm{~km}$ hacia el oeste de Jaizkibel, es claramente migrador; A. Galarza, com. pers.).

Carduelis chloris (L., 1758). Cría en arbolado, en campiñas y ecotonos de bosque de caducifolios (Aierbe et al., 2001). Migrante presahariano.

Carduelis carduelis (L., 1758). Cría en arbolado, en campiñas y ecotonos de bosque de caducifolios, también en árboles dispersos en argomales, brezales, etc. (Aierbe et al., 2001). Posiblemente sedentario.

Carduelis cannabina (L., 1758). Cría en arbustos, en brezales, manchas de argomal y campiña (Aierbe et al., 2001). En teoría sedentario (Cramp \& Perrins, 1994a), pero no comprobado.

Pyrrhula pyrrhula (L., 1758). Cría en arbolado de caducifolios. Citado en un jardín en el núcleo urbano de Hondarribia (Aierbe et al., 2001). En la zona del golf es habitual y en años se anilla regularmente durante la época de cría (A. Mendiburu). Sedentario.

Coccothraustes coccothraustes (L., 1758). Se ha citado como reproductor en el golf. En 2001 se localizó una pareja con pollos volanderos (J. Belzunce, com. per.). Sedentario, aunque no comprobado.

Emberiza citrinella L., 1758. Ligado a manchas de argomal, brezales, etc. (Aierbe et al., 2001). En teoría sedentario (Cramp \& Perrins, 1994b), pero no comprobado.

Emberiza cirlus L., 1766. Citado en manchas de argomal y brezales (Aierbe et al., 2001). Cría alguna pareja en la ladera que da al mar (J. Belzunce, com. per.). Sedentario.

Emberiza cia L., 1766. Cría en general en argomal y brezal (Aierbe et al., 2001). Sedentario.

\section{DISCUSIÓN}

Se constata en Jaizkibel y Jaizubia la nidificación, continua o esporádica, entre 1999 y 2012, de 81 especies de aves, lo cual supone un $64,8 \%$ de las especies que se citan para toda la provincia de Gipuzkoa, de acuerdo al último Atlas (Aierbe et al., 2001). En cuanto a especies interesantes desde un punto de vista de la conservación, se detectan 16 especies incluidas en el Catálogo Vasco de Especies Amenazadas.

Un primer punto a tener en cuenta es que la revisión aquí desarrollada se basa en un esfuerzo de muestreo bajo y heterogéneo. Así, el único censo completo para la zona se desarrolló para llevar a cabo el atlas de aves nidificantes de Gipuzkoa (Aierbe et al., 2001), mientras que el resto de citas provienen todas de censos parciales o puntuales y de observaciones casuales. En consecuencia, una prospección más meticulosa, específica para Jaizkibel, podría resultar en un incremento en el número de especies nidificantes detectadas (Magurran, 1989). Asimismo, harían falta censos de carácter cuantitativo para determinar la densidad de las poblaciones que se reproducen en la zona, e.g., a través de transectos o estaciones de escucha (Carrascal \& Palomino, 2008).
Globalmente, la riqueza de especies de aves nidificantes en el área de estudio es alta, probablemente una de las más elevadas de Gipuzkoa para el área de muestreo considerada. Esto podría ser debido, principalmente, a la heterogeneidad de hábitats de Jaizkibel, que incluyen roquedos, landas, formaciones arbustivas y forestales, humedales. La recuperación de hábitat potencial natural en el futuro (e.g. robledal), enriquecería la diversidad de la avifauna reproductora en Jaizkibel, o como mínimo consolidaría aquellas especies que, por depender de los hábitats más amenazados, son hoy muy escasas o incluso no llegan a reproducirse de manera continuada. No obstante, la existencia de etapas de vegetación anteriores al bosque (en este caso robledal), como son los pastos, brezales y parches de argomal es determinante para determinadas especies, como es el caso de determinados fringílidos y emberícidos. Igualmente, el paisaje de campiña es clave para otras especies, entre ellas Lanius collurio, cada vez más raro en la zona y a escala continental (Tucker \& Heath, 2004).

Por otro lado, nótese que se han incluido en el área de muestreo las marismas de Jaizubia y humedales asociados, que aportan una gran cantidad de especies estrictamente asociadas a humedales. La inclusión de Jaizubia en el área de muestreo no debe considerarse como un defecto del estudio, pues este humedal depende, en gran medida y a diferencia de otras zonas húmedas del entorno, de las aguas que proceden directamente de la ladera septentrional de Jaizkibel. Además, varias especies que durante el periodo de cría ocupan las laderas del monte, se desplazan tras la cría, principalmente en invierno, a la marisma de Jaizubia. Un caso típico de este comportamiento es el de Cisticola juncidis.

En cuanto a especies prioritarias para la conservación, cabría destacar Neophron percnopterus y Charadrius dubius. La primera especie es escasa en Gipuzkoa, con 10 parejas citadas para todo el territorio (Álvarez et al., 2009). La nidificación en Jaizkibel refuerza el crecimiento poblacional de la especie observado durante los últimos años en Gipuzkoa (Álvarez et al., 2009), suponiendo, además, la colonización de un sector, el NE de Gipuzkoa, donde hasta ahora no criaba la especie. Neophron percnopterus está catalogada "En Peligro" a escala peninsular (Madroño et al., 2004) y, en consecuencia, es una especie con un interés máximo desde el punto de vista de la conservación. En este contexto, la conservación y protección de la zona de cría en Jaizkibel y el hábitat del entorno es fundamental para garantizar su permanencia. Charadrius dubius es, asimismo, una especie muy escasa en Gipuzkoa y en la CAPV. Su nidificación en el caso de Jaizkibel se circunscribe a la marisma de Jaizubia.

En conclusión, Jaizkibel y la regata de Jaizubia son ricos en avifauna reproductora, llegando a acoger especies prioritarias para la conservación. Para realizar un inventario más exacto habría que planificar censos a través de un diseño de muestreo por estratos y específico para la zona, en el que, además, se contemplaran estimaciones de carácter cuantitativo (e.g., Carrascal \& Palomino, 2008). 


\section{AGRADECIMIENTOS}

Agradezco a las persones que, muy amablemente, contribuyeron a la aportación de citas: T. Aierbe, J. Belzunce, D. Calleja, M. Guereñu, A. Herrero, J. Múgica, M. Olano. K. Álvarez y A. Galarza ayudaron con sus valiosos comentarios a mejorar una primera versión del trabajo.

\section{BIBLIOGRAFÍA}

Aierbe, T., Olano, M., Vázquez, J. 2001. Atlas de las aves nidificantes de Gipuzkoa. Munibe, Cienc. Nat. 52: 5-136.

Álvarez, F., Olano, M., Aierbe, T., Vázquez, J., Izkeaga, P., Ugarte, J. 2005. Población, distribución y reproducción del Halcón peregrino (Falco peregrinus) en Gipuzkoa (2003-2004). Munibe, Cienc. Nat. 56: 113-132.

Álvarez, F., Ugarte, J., Vázquez, J., Aierbe, T., Olano, J. M. 2009. Distribución y reproducción del Alimoche común (Neophron percnopterus) en Gipuzkoa. Munibe, Cienc. Nat. 57: 213-224.

Arizaga, J., Galarza, A., Herrero, A., Hidalgo, J., Aldalur, A. 2009. Distribución y tamaño de la población de la Gaviota Patiamarilla Larus michahellis lusitanius en el País Vasco: tres décadas de estudio. Rev. Catalana d'Ornitologia 25: 32-42.

Arizaga, J. (Ed.) 2012. Jaizkibel, una cápsula del tiempo para el patrimonio natural y cultural (Simposio). Sociedad de Ciencias Aranzadi. Donostia-San Sebastián.

Carrascal, L. M., Palomino, D. 2008. Las aves comunes reproductoras en España. Población en 2004-2006. SEO/BirdLife. Madrid.
Clavell, J., Copete, J. L., Gutiérrez, R., de Juana, E., Lorenzo, J. A. 2005. Lista de las aves de España. SEO/BirdLife. Madrid.

Cramp, S., Perrins, C. M. 1994a. Handbook of the Birds of Europe, the Middle East and North Africa. Vol. 8. Oxford University Press. Oxford.

Cramp, S., Perrins, C. M. 1994b. Handbook of the Birds of Europe, the Middle East and North Africa. Vol. 9. Oxford University Press. Oxford

Madroño, A., González, C., Atienza, J. C. 2004. Libro Rojo de las Aves de España. DGB-SEO/BirdLife. Madrid.

Magurran, A. 1989. Diversidad ecológica y su medición. Vedrá Barcelona.

Mendiburu, A., Aranguren, I., Elosegi, Z., Jauregi, J. I., Sánchez, J. M., Cuadrado, J. F., Alonso, D., Arizaga, J. 2009. Resultados de la primera campaña de anillamiento en el paso migratorio posnupcial en la vega de la regata de Jaizubia (marismas de Txingudi). Rev. Anill. 23: 26-34.

Mendiburu, A., Sánchez, J. M., Jauregi, J. I., Arizaga, J. 2010 Estructura y dinámica de la comunidad invernante de paseriformes y afines en el carrizal de Jaizubia (marismas de Txingudi, Gipuzkoa). Munibe, Cienc. Nat. 58: 173-185.

Tellería, J.L., Asensio, B., Díaz, M. 1999. Aves Ibéricas. II. Paseriformes. J. M. Reyero Editor. Madrid.

Tucker, G. M., Heath, M. F. 2004. Birds in Europe: population estimates, trends and conservation status. BirdLife International. Cambridge. 